\title{
Mechanisms for concentrating Rho1 during cytokinesis
}

\author{
Satoshi Yoshida, Sara Bartolini, and David Pellman ${ }^{1}$ \\ Department of Pediatric Oncology, Dana-Farber Cancer Institute, Harvard Medical School, Boston, Massachusetts 02115, USA; \\ Division of Hematology/Oncology, Children's Hospital Boston, Boston, Massachusetts 02115, USA; and Department of Cell \\ Biology, Harvard Medical School, Howard Hughes Medical Institute, Boston, Massachusetts 02115, USA
}

\begin{abstract}
The small GTP-binding protein, Rho1/RhoA plays a central role in cytokinetic actomyosin ring (CAR) assembly and cytokinesis. Concentration of Rho proteins at the division site is a general feature of cytokinesis, yet the mechanisms for recruiting Rho to the division site for cytokinesis remain poorly understood. We find that budding yeast utilizes two mechanisms to concentrate Rho1 at the division site. During anaphase, the primary mechanism for recruiting Rho1 is binding to its guanine nucleotide exchange factors (GEFs). GEF-dependent recruitment requires that Rho1 has the ability to pass through its GDP or unliganded state prior to being GTP-loaded. We were able to test this model by generating viable yeast lacking all identifiable Rho1 GEFs. Later, during septation and abscission, a second GEF-independent mechanism contributes to Rho1 bud neck targeting. This GEF-independent mechanism requires the Rho1 polybasic sequence that binds to acidic phospholipids, including phosphatidylinositol 4,5-bisphosphate (PIP2). This latter mechanism is functionally important because Rho1 activation or increased cellular levels of PIP2 promote cytokinesis in the absence of a contractile ring. These findings comprehensively define the targeting mechanisms of Rho1 essential for cytokinesis in yeast, and are likely to be relevant to cytokinesis in other organisms.
\end{abstract}

[Keywords: Rho1; cytokinesis; GEF; polybasic sequence; yeast]

Supplemental material is available at http://www.genesdev.org.

Received January 26, 2009; revised version accepted February 24, 2009.

Cytokinesis partitions the genetic material between daughter cells. In most eukaryotes, cytokinesis is accomplished by constriction of a cytokinetic actomyosin ring (CAR) and targeted membrane deposition (Balasubramanian et al. 2004; Barr and Gruneberg 2007). Cytokinesis failure causes polyploidization and genetic instability, which can lead to tumorigenesis (Fujiwara et al. 2005). It is thus critical that there be tight spatial and temporal controls that coordinate cytokinesis with other events during cell division.

In animal and yeasts, a small Rho family GTPase (RhoA in humans and Rhol in budding yeast) is a key regulator of cytokinesis (Balasubramanian et al. 2004; Barr and Gruneberg 2007). Rho1/RhoA has clear roles in CAR assembly, at least in part by the activation of actin nucleators/assembly factors called formins. RhoA also promotes myosin II contractility. Myosin II drives CAR constriction, the flow of contractile elements into the cell equator, traction forces, and anaphase cell shape changes, all of which can contribute to cytokinesis (Piekny et al. 2005).

${ }^{1}$ Corresponding author.

E-MAIL david_pellman@dfci.harvard.edu; FAX (617) 632-3352.

Article is online at http://www.genesdev.org/cgi/doi/10.1101/gad.1785209.
Like all regulatory GTPases, Rho proteins are activated by GTP loading, which in cells is triggered by guanine nucleotide exchange factors (GEFs). Rho proteins are inactivated by GTP hydrolysis, which is accelerated by GTPase-activating proteins (GAPs). Guanine nucleotide dissociation inhibitors (GDIs) also inhibit Rho activation by preventing GDP release from Rho and by sequestering Rho proteins from membranes (Rossman et al. 2005). Rho family GTPases do not always function as simple on-off switches where the GTP-bound form is "on" and the GDPbound form is "off"; for some processes, Rho GTPases must cycle between the GTP and GDP-liganded states (Symons and Settleman 2000).

Recently there has been considerable progress in defining the temporal regulation of Rho during mitosis. In human and yeast cells, the total cellular amount of RhoGTP significantly increases during anaphase (Kimura et al. 2000; Yoshizaki et al. 2003; Yoshida et al. 2006; Kono et al. 2008). In budding yeast, the Polo-like kinase Cdc5 triggers Rhol activation, increasing both the total cellular concentration of Rhol-GTP and triggering the recruitment and local activation of Rhol at the division site (Yoshida et al. 2006). These effects are mediated at least in part by Cdc5/Polo phosphorylation of Rhol GEFs, which, when phosphorylated, are targeted to the division 
site. If Rhol GEFs fail to be recruited to the division site, Rhol itself is neither activated nor concentrated at the division site. Although there are interesting and important mechanistic differences in how yeast and animal cells recruit Rho GEFs to the division site, it is now known that a similar Polo-RhoGEF-Rho regulatory module triggers CAR assembly in animal cells (Takaki et al. 2008). In human cells, the Polo-like kinase Plk1 is necessary to target the RhoGEF Ect2 to the cell equator, which in turn promotes the accumulation of active RhoA, CAR assembly, and CAR constriction. Plk1 also appears to be necessary for the increase in total cellular RhoA-GTP during late mitosis (Takaki et al. 2008).

Although there has been recent progress linking cell cycle signals to Rho activation, the spatial control of Rho during cytokinesis, particularly its underlying molecular mechanism, is not well understood (Yoshizaki et al. 2003; Bement et al. 2005). Before being concentrated at the cell equator, Rho proteins must be delivered to the plasma membrane. Like Ras proteins, Rho GTPases are synthesized in the cytoplasm. After synthesis, Rho GTPases undergo post-translational modifications of a C-terminal CAAX prenylation motif on the membrane of the endoplasmic reticulum. This single prenylation is necessary but not sufficient for localization of Rho-GTPases to the plasma membrane. Plasma membrane targeting requires either that the G-protein undergo lipid modifications at additional sites or that the G-protein contain a polybasic sequence (PBS) adjacent to the CAAX motif (Heo et al. 2006). The positively charged amino acid residues in the PBS promote plasma membrane localization via electrostatic interactions with negatively charged phospholipids in the plasma membrane such as phosphatidylinositol 4,5-bisphosphate (hereafter referred to as PIP2) or phosphatidylinositol 3,4,5-trisphosphate (which is present in animal cells but not in budding yeast).

Budding yeast Rhol, like mammalian RhoA, is singly prenylated and contains a PBS. Bulk Rhol is delivered to the plasma membrane through the secretory system (Abe et al. 2003). However, secretion is not required for CAR assembly and directed secretion to the division site occurs only after mitotic exit (Dobbelaere and Barral 2004; VerPlank and Li 2005; Zhang et al. 2006). Thus, polarized secretion cannot explain the concentration of Rhol at the division site during anaphase when the CAR is assembled.

In various organisms, the concentration of RhoA/Rhol at the division site requires Rho GEFs but several underlying mechanisms might explain this requirement. One model is that Rho GEFs might directly recruit Rho proteins to the division site. This could be mediated by interactions with the catalytic Dbl homology (DH) domain or, by other modular domains contained within Rho GEFs. For example, budding yeast Rhol GEFs contain a CNH (Citron-Nik1 homology) domain, which has been implicated in G-protein binding (Taira et al. 2004). Alternatively, Rho GEFs could be important for achieving a threshold level of Rho GTP loading that is required for further concentration and maintenance of Rho at the division site (Bement et al. 2006).
Here, we use budding yeast to comprehensively define the mechanisms that concentrate Rhol at the division site to enable cytokinesis. Two temporally distinct mechanisms concentrate Rhol at the bud neck. During CAR assembly (anaphase), Rhol is targeted to the division site by its GEFs. Rho1 recruitment and its subsequent function in CAR assembly requires the catalytic activity of the GEFs and Rho1's ability to pass through a GDP or unliganded state. After mitotic exit and CAR starts constriction, a second GEF-independent mechanism can concentrate Rhol in the membrane microdomain that is formed by the split septin rings. This mechanism requires the Rho1 PBS, likely involves interactions with PIP2, and is independent of the Rhol nucleotide-bound state. Finally, genetic experiments demonstrate a functional role for Rhol and phospholipids during the late stages of cytokinesis. Clearly separating these two roles of Rhol in cytokinesis was feasible because in most budding yeast strains the CAR is not essential, enabling direct study of late-stage CAR-independent mechanisms (Vallen et al. 2000). Together, these findings identify novel mechanisms of Rho regulation that are likely to be conserved.

\section{Results}

\section{GTP-locked Rho1 cannot promote CAR assembly}

Because of the conserved role of Polo kinases in controlling global and local Rhol/RhoA activation during anaphase, we determined if a "constitutively active" GTPlocked Rhol variant would bypass the requirement of $\mathrm{Cdc5} /$ Polo to assemble cytokinetic actin rings. GTPhydrolysis-defective mutants (Rhol-G19V and Rho1Q68H) were expressed at endogenous levels in cdc5-2 strains and CAR assembly was visualized. These Rhol mutants were previously shown to behave as constitutively active and to be hyperactive for several Rhol functions (Delley and Hall 1999; Sekiya-Kawasaki et al. 2002; Abe et al. 2003; Valdivia and Schekman 2003). Unexpectedly, we found that neither of these GTP-locked Rhol mutants rescued the CAR assembly defect of $c d c 5$ 2 cells (Fig. 1A; Supplemental Fig. S1). We also generated "rapid cycling" mutants, Rho1-C25A and Rho1-F35L. The analogous mutations in human Cdc42 (Cdc42C18A and Cdc42-F28L) have a high spontaneous nucleotide dissociation rate that leads to rapid exchange of GDP for GTP even in the absence of GEFs (Lin et al. 1997; Rossman et al. 2002). These rapid-cycling versions of Rhol were indeed hyperactive in yeast and rescued the lethality caused by the deletion of the three Rhol GEFs (see below). However, like the GTP-locked mutants, Rho1-C25A and Rho1-F35L also failed to rescue the CAR assembly defect of $c d c 5-2$ cells (Supplemental Fig. S1). Importantly, control experiments demonstrated that neither the GTP-locked Rhol mutants nor the rapid cycling mutants interfered with normal CAR assembly in $c d c 15-2$ cells (Fig. 1A; Supplemental Fig. S1). cdc15-2 cells arrest in telophase with active Cdc5/Polo; these cells recruit the Rho1GEF Tus1 to the bud neck and assemble normal appearing contractile rings (Supplemental 
Yoshida et al.

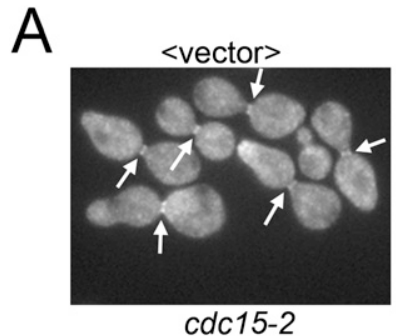

cdc15-2

B
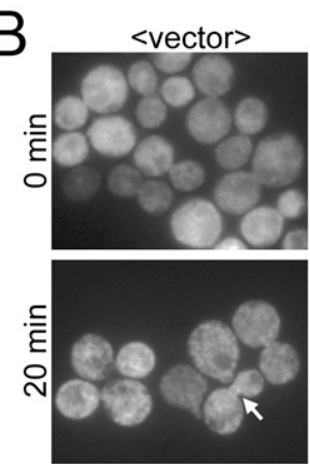

( $\Delta c d c 20$, GALL-CDC20, $\Delta r h 01$, rho1-2, TPM2-GFP)

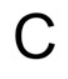

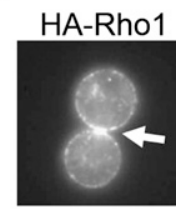

cdc15-2
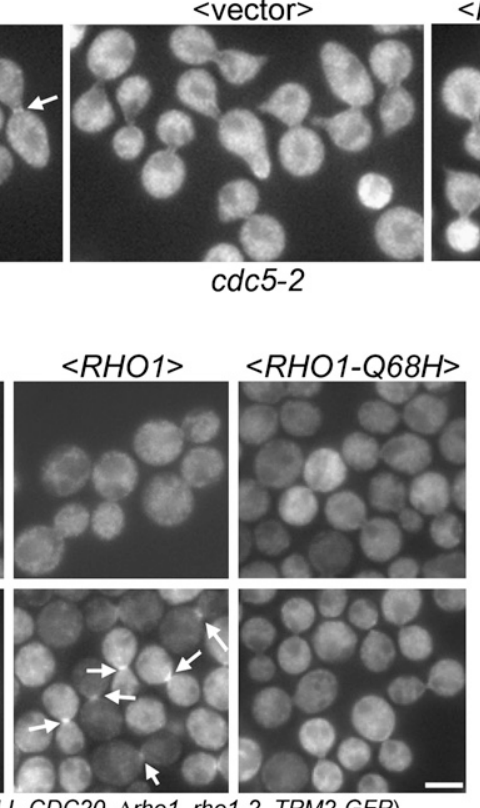

cdc5-2

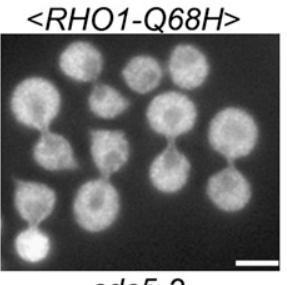

cdc5-2
$\%$ CAR assembly

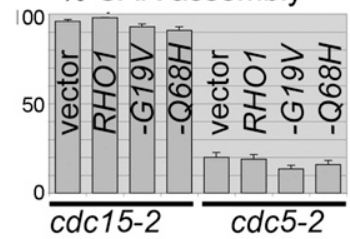

rho1-2 inactivation

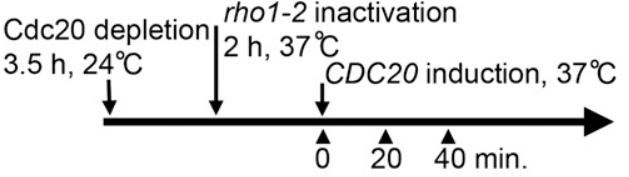

$\%$ CAR assembly

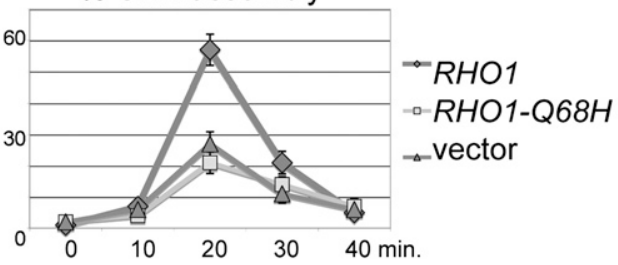

$\mathrm{D}$

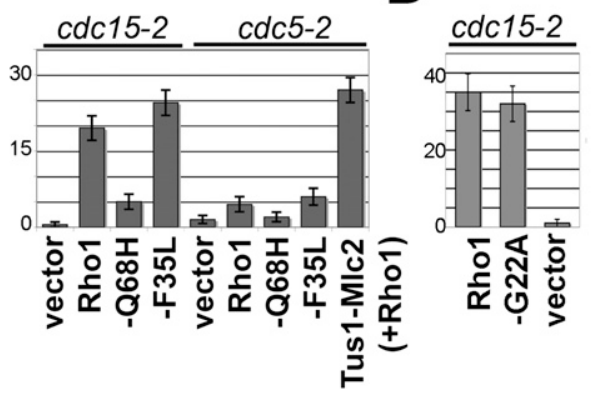

Figure 1. GTP-locked Rhol fails to support CAR assembly because it fails to be targeted to the bud neck during anaphase. $(A)$ GTPlocked Rhol cannot bypass the requirement for Polo kinase to promote CAR assembly. Control cdc15 RHO1strains (telophase arrest, high Cdc5/Polo activity) or cdc5 RHO1 strains (telophase arrest, low Cdc5/Polo activity) bearing the indicated plasmids were released from a G1 block for $2 \mathrm{~h}$ at $34.5^{\circ} \mathrm{C}$ and CAR assembly was monitored with Tpm2-GFP. (B) RHO1-Q68H (GTP-locked allele) is a recessive loss-of-function allele with respect to CAR assembly. The indicated strains were synchronized in metaphase by Cdc20 depletion, released under conditions that inactivate endogenous Rhol, and CAR assembly was monitored at intervals by Tpm2-GFP. The RHO1 alleles are expressed from the endogenous promoter on low-copy-number plasmids. (C) GTP-locked Rhol fails to concentrate at the bud neck during anaphase. Cells were arrested in telophase with high or low Cdc5/Polo activity as in $A$. The cells either expressed the wildtype Rho1 GEF Tus1 or a Tus1-Mlc2 fusion that forces Tus1 localization to the bud neck. The localization of HA-tagged wild-type Rho1 or the indicated Rhol mutants was determined by immunofluorescence. $(D)$ Nucleotide free Rho1-G22A localizes to the division site. Because Rho1-G22A acts as a dominant-negative mutant and is highly toxic when expressed even from the endogenous promoter (not shown), this experiment was performed in strains containing activated Pkc1, Pkc1-R398P (Nonaka et al. 1995), which we found rescued the near-lethal phenotype of Rho1-G22A expression. Arrows indicate the bud neck localization of Tpm2 $(A, B)$ or Rhol $(C)$. Arrowheads in $C$ indicate lack of the bud neck signal. Error bar is the SEM. Bar, $3 \mu \mathrm{m}$.

Fig. S1; Yoshida et al. 2006). Thus, mutational activation of Rhol is not sufficient to promote CAR assembly in the absence of functional Cdc5/Polo.

Next, we determined if GTP-locked Rhol could substitute for endogenous Rhol in cells progressing through the cell cycle. To study CAR assembly independently of the role of Rhol early in the cell cycle, rho1-2 cells were synchronized in metaphase by depletion of anaphase promoting complex regulator Cdc20. Rhol was then inactivated by shifting the temperature to $37^{\circ} \mathrm{C}$. Cells were released from the metaphase block and CAR assembly was visualized. The sharp cell synchrony achieved in this experiment revealed a clear requirement for Rhol in CAR assembly, extending the previous findings of Tolliday et al. (2003). Although wild-type Rhol fully complemented the CAR assembly defect in this strain, GTP-locked Rhol failed to do so (Fig. 1B). This finding could not be explained by a defect in cell cycle 
progression because anaphase entry occurred on schedule in GTP-locked Rhol-expressing cells (data not shown). Together, these results suggest that GTP-locked Rhol behaves as a loss-of-function mutant with respect to CAR assembly.

Next we determined whether the inability of "activated" Rhol variants to trigger CAR assembly could be explained by a defect in their ability to concentrate at the division site. In cdc15-2-arrested cells, wild-type Rhol and Rho1-F35L properly localized to the bud neck (Fig. 1C). In contrast, GTP-locked (Rho1-Q68H) failed to do so (Fig. 1C). An appealing explanation for this result would be that Rhol is recruited to the bud neck by interaction with its GEFs (during anaphase, primarily Tus1), whose catalytic domains bind specifically to GDP-liganded or nucleotide free Rhol and thus do not bind GTP-locked mutants (Schmelzle et al. 2002). Consistent with this hypothesis, a nucleotide-free Rhol variant (Rho1-G22A) (Schmelzle et al. 2002), was targeted as efficiently as wild-type Rhol to the division site neck in cdc15-2-arrested cells (Fig. 1D).

Further support for the idea that Rhol must pass through the GDP or unliganded state to enable CAR assembly came from characterizing cells lacking Cdc5/ Polo where Rho GEFs are not targeted to the division site. Like wild-type Rho1, neither Rho1-Q68H (GTPfixed) nor Rho1-F35L (rapid-cycling) concentrated at the bud neck in cdc5-2 cells (Fig. 1C). In contrast, if the Rho-GEF Tus1 is artificially tethered to the bud neck by fusion to a nonessential myosin light chain (the Tus1Mlc2 fusion protein described in Yoshida et al. [2006]), the bud neck concentration of Rhol and CAR assembly can be restored in Cdc5/Polo-deficient cells (Fig. 1C; Supplemental Fig. S1).

Together, these results suggest that Rhol must be able to pass through the GDP or unliganded state in order to be concentrated at the division site. The loss-of-function effect was unexpected given that GTP-locked Rhol is known to be activated for several Rhol functions in vivo and to display enhanced affinity for all the known Rhol effectors in vitro (Park and Bi 2007). However, the failure of GTP-locked Rhol to support CAR assembly suggests that nucleotide cycling of Rhol is critical for cytokinesis.

\section{Viable yeast strains lacking all Rho1 GEFs}

The hypothesis that RhoGEFs recruit Rhol to the division site via their catalytic mechanism would most simply be tested by studying cells lacking RhoGEFs or, more specifically, cells lacking the catalytic function of RhoGEFs. However, this is challenging to accomplish because Rhol GEF function is essential for cell viability. To circumvent this difficulty we sought to engineer viable yeast lacking all recognizable Rhol GEFs.

The budding yeast genome encodes four DH domaincontaining RhoGEFs, Cdc24, Rom1, Rom2, and Tus1 (Fig. 2A; Park and Bi 2007). Cdc24 is specific to the polarityregulating GTPase Cdc42; the other three are specific regulators of Rho1. We constructed strains with complete deletions of the three Rhol GEF genes (hereafter referred
A

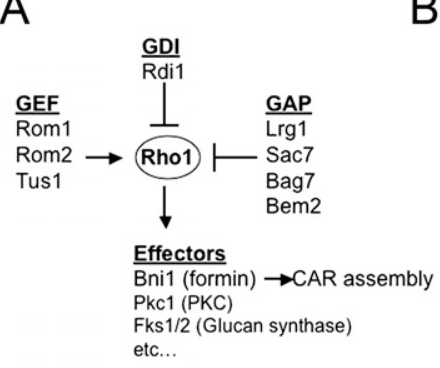

B $\langle R O M 2><r o m 2-T 666 A>$
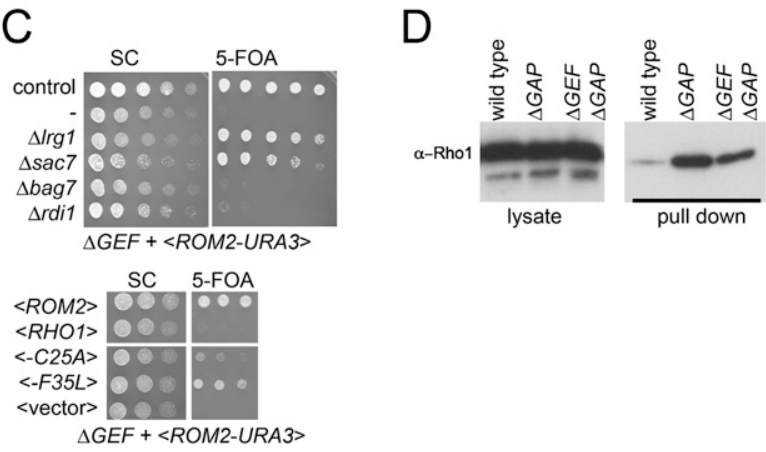

Figure 2. Creation of viable yeast strains lacking all Rho1 GEFs. (A) Outline of Rhol regulation in budding yeast. See the text for details. (B) A plasmid shuffle assay demonstrates that the essential function of Rhol GEFs requires their catalytic activity. The $\triangle G E F$ strain grows in the presence of a $<R O M 2$ URA3 CEN> plasmid but fails to grow after counterselection with 5-FOA. A point mutation in a critical catalytic residue in the DH domain of Rom2 (T666A) fails to complement the lethality of the $\triangle G E F$ strain. The indicated strains were grown for $3 \mathrm{~d}$ at $24^{\circ} \mathrm{C}$. (C) GAP gene deletions or rapid cycling Rhol mutants restore viability to the $\triangle G E F$ strain. A plasmid shuffle was performed to assess viability as in $B$. (Top) Deletion of the Rho1 GAP genes $L R G 1$ or $S A C 7$ (but not $B A G 7$ ) restored growth to the $\triangle G E F$ strain at $24^{\circ} \mathrm{C}$. Deletion of the sole Rhol GDI gene RDI1 had no effect on growth of the $\triangle G E F$ strain. (Bottom) Two predicted Rhol "rapid cycling" mutations restore growth to the $\triangle G E F$ strain. $(D)$ The $\triangle G E F \triangle G A P$ strain has high levels of RholGTP; deletion of Rhol GEFs has only a subtle effect on the total cellular levels of Rho1-GTP. GTP-Rhol was assayed in the indicated strains by a Rho-binding domain pull-down assay.

to as the $\triangle G E F$ strain). As expected, the $\triangle G E F$ strain was inviable (Fig. 2B,C). The lethality was due to the absence of exchange activity toward Rhol because expression of Rom2-T666A, a mutant in the conserved catalytic residue of $\mathrm{DH}$ domain (Zhu et al. 2000) failed to rescue the lethality (Fig. 2B).

Strikingly, the lethality of the $\triangle G E F$ strain could be bypassed either by deletion of one of the two major Rho1 GAPs Lrg1 or Sac7 (Fig. 2A,C; Lorberg et al. 2001; Watanabe et al. 2001; A. Schmidt et al. 2002), or by expression of the rapid cycling Rhol variants (Fig. 2C). Although these strains grew at $24^{\circ} \mathrm{C}$, they exhibited phenotypes suggesting abnormal Rhol signaling: They failed to grow at $37^{\circ} \mathrm{C}$ and were supersensitive to cell wall-damaging agents (data not shown). Similar growth defects were observed in $\triangle G E F$ strains rescued by the 
rapid cycling variant Rho1-F35L mutant. Consistent with other genetic data suggesting that Bem 2 and Bag7 are not major Rhol GAPs (A. Schmidt et al. 2002; Knaus et al. 2007), deletion of a potential Rho1 GAP Bem2 causes synthetic sickness with deletion of the Rho1 GEF Rom2 (Manning et al. 1997) and deletion of the putative Rhol GAP Bag7 failed to rescue the $\triangle G E F$ strain (Fig. 2C). Furthermore, deletion of the only yeast RhoGDI, Rdil did not rescue the lethality of $\triangle G E F$ cells (Fig. 2C). Because the $\Delta$ rho1gef $\Delta \operatorname{lrg} 1$ grew better than $\Delta$ rho1gef $\Delta$ sac7, subsequent experiments were performed with $\Delta$ rho1gef $\triangle \operatorname{lrg} 1$ (hereafter referred to as $\triangle G E F \triangle G A P$ ).

Next we examined the impact of GEF deletion on the total cellular level of GTP-loaded Rho1. By affinity with the Rho-binding domain of protein kinase $\mathrm{C}$, the control $\Delta \operatorname{lrg} 1$ cells had significantly increased levels of Rho1GTP. Quite surprisingly, the $\triangle G E F \triangle G A P$ cells had significantly higher levels of Rhol-GTP than wild-type cells and only slightly lower levels than measured in the $\Delta \operatorname{lrg} 1$ cells (Fig. 2D). Thus, in budding yeast, GAPs, not GEFs, play the major role in setting the total cellular concentration of GTP-loaded Rhol.

The catalytic activity of Rho1 GEFs is required to concentrate Rho1 at the bud neck during anaphase

$\triangle G E F \triangle G A P$ cells failed to assemble normal contractile actomyosin rings and exhibited striking defects in cytokinesis. In wild-type and $\Delta \operatorname{lrg} 1$ strains, late anaphase cells contained clear, sharp labeling of the contractile ring at the mother-bud neck. In contrast, $\triangle G E F \triangle G A P$ strains assembled markedly fewer CARs (Fig. 3A,B), and even when assembled, the CAR was faint and/or disorganized. Consistent with this CAR assembly defect, $\triangle G E F \triangle G A P$ strains were obviously defective in cytokinesis; $\triangle G E F$ $\triangle G A P$ cells formed clumps with multiple connected cells (Supplemental Fig. S2A). Finally, $\triangle G E F \triangle G A P$ cells were defective in concentrating Rhol at the bud neck, particularly during anaphase (see below).

We next defined the Rhol GEFs that could restore CAR assembly to $\triangle G E F \triangle G A P$ cells and then tested whether CAR assembly specifically requires the $\mathrm{DH}$ domains of these Rhol GEFs. Our previous results suggested that Tus1 is the major Rhol GEF required for CAR assembly; Rom2 plays a partially overlapping, but more minor role (Yoshida et al. 2006). Furthermore, Rom2 does not become detectably concentrated at the neck until late cytokinesis, after septin ring splitting and mitotic exit (Yoshida et al. 2006). A third Rhol GEF, Rom1, appears to play little or no role in CAR assembly (Yoshida et al. 2006). Consistent with these results, we found that Tus1 largely restored CAR assembly in $\triangle G E F \triangle G A P$ cells and that Rom 2 had little detectable impact on CAR assembly (Fig. 3C). Importantly, the ability of Tus 1 to restore CAR assembly required a functional catalytic domain: Neither a Tus1 DH domain deletion (Tus1- $\Delta \mathrm{DH}$ ) nor a Rom2 DH domain point mutant (Rom2-T666A) were able to restore CAR assembly in the $\triangle G E F \triangle G A P$ strain (Fig. 3C). In addition, neither Tus1- $\Delta \mathrm{DH}$ nor Rom2-T666A were able to restore Rhol targeting in $\triangle G E F \triangle G A P$ cells (Fig. $3 \mathrm{D}$ ).
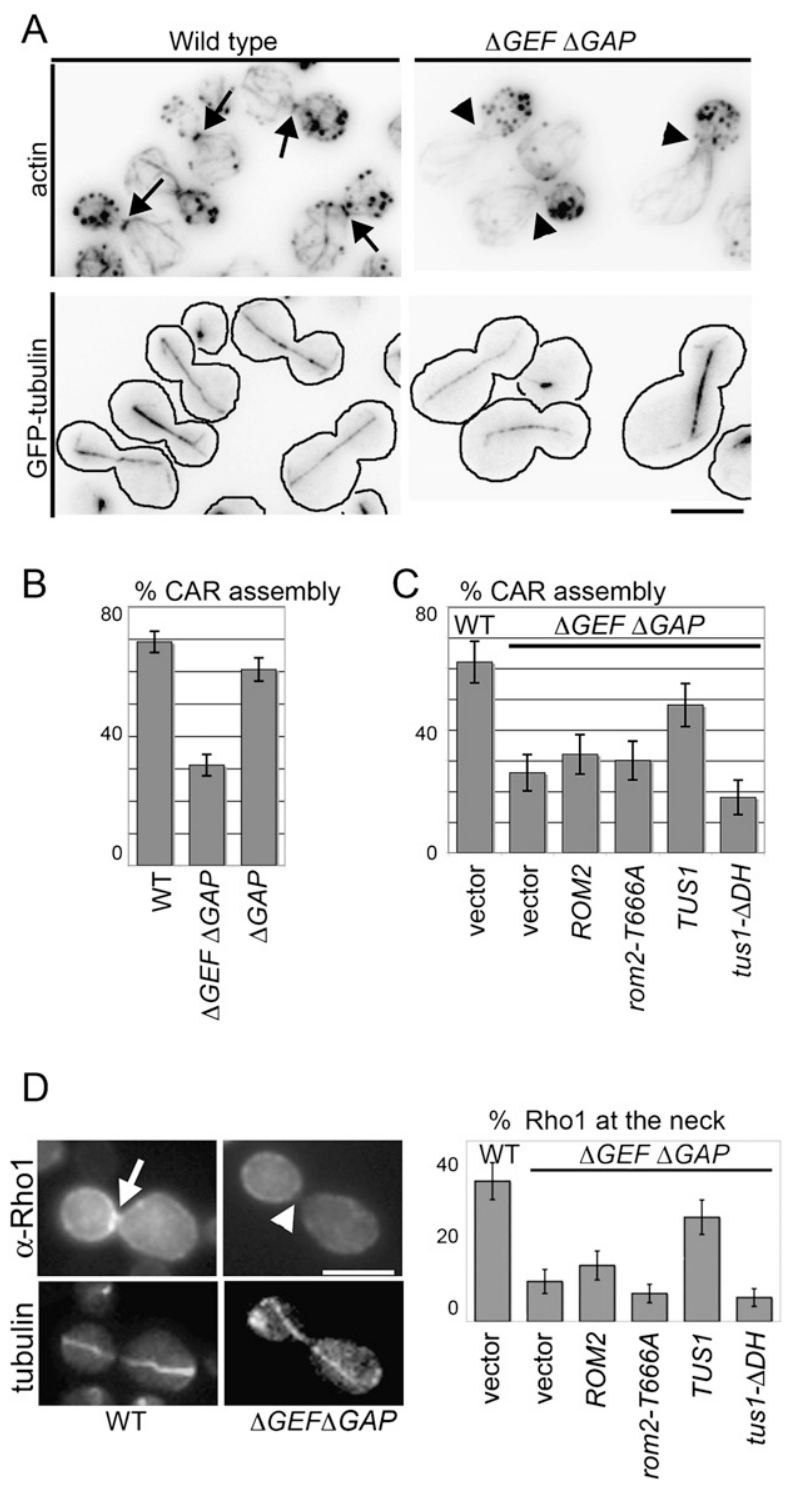

Figure 3. The catalytic activity of Rhol GEFs is required for CAR assembly. $(A)$ Defective CAR assembly in the absence of catalytically active Rhol GEFs. (Top panel) Alexa 568-phalloidin labeling to visualize the CAR in wild-type and $\triangle G E F \triangle G A P$ cells. (Bottom panel) Anaphase spindles visualized with GFPtubulin. Arrows indicate the position of the CAR, and arrowheads indicate absence of the CAR. $(B, C)$ Percentage of anaphase cells (fully elongated spindles) with detectable CARs in the indicated strains. $(D)$ Localization of Rhol in anaphase cells lacking Rhol GEFs. Rhol was detected by immunofluorescence using a rabbit anti-Rhol antibody in the indicated strains. Microtubules were labeled with GFP- $\alpha$-tubulin. Representative cells are shown in the left and the results are summarized in the right. The arrow indicates bud neck localizaton of Rhol, and the arrowhead indicates the absence of Rhol.

Both Rom2-T666A and Tus1- $\Delta \mathrm{DH}$ were expressed at comparable steady state levels relative to the wild-type controls (Supplemental Fig. S2B). Thus, the nucleotide exchange activity of GEFs is required for the anaphasespecific concentration of Rhol at the bud neck. 
Live cell imaging reveals sequentially acting mechanisms that concentrate Rho1 at the division site

To characterize the behavior of Rhol in living cells, we generated a GFP-RHO1 fusion expressed at endogenous levels from the native promoter. GFP-RHO1 complements a rho1-null strain at $24^{\circ} \mathrm{C}$ (Supplemental Fig. S3A) and has no detectable defect in cell morphology or cytokinesis. All of the experiments reported below were performed at $24^{\circ} \mathrm{C}$ because GFP-Rhol becomes unstable at $37^{\circ} \mathrm{C}$ (Supplemental Fig. S3B). To our knowledge this is the first GFP-Rhol fusion that can functionally replace it's endogenous counterpart. The localization of GFP-Rhol was generally consistent with the localization of Rhol determined previously by immunofluorescence (Yamochi et al. 1994; Ayscough et al. 1999; Abe et al. 2003). However, GFP-Rhol enabled us to visualize an endomembrane/vacuolar pool of Rhol (Supplemental Fig. S3C) that was detected by biochemical experiments but not previously visualized (Eitzen et al. 2001; Paumi et al. 2007). We constructed GTP-locked and rapid cycling variants of GFP-Rhol. Examination of these mutants was consistent with our immunofluorescence results (Fig. 3D) and further supported the GEF-dependent recruitment of Rhol during anaphase (Fig. 4A). Consisistent with the requirement of nucleotide exchange, GTP-locked Rho1-Q68H failed to localize to the bud neck in anaphase regardless of the presence or absence of GEFs (Fig. 4A). Note that GTP-locked Rhol accumulates in the bud prior to mitotic exit, presumably because Rhol is delivered to the cell cortex via polarized secretion.

GFP-Rhol enabled us to precisely characterize the timing of Rhol recruitment to the division site at endogenous levels in living cells. Consistent with its requirement for CAR assembly (CAR assembles shortly before mitotic exit) (Bi et al. 1998; Tolliday et al. 2003), GFP-Rhol was detectably concentrated at the bud neck 3-4 $\min (n=14)$ prior to mitotic exit (Fig. 4B). This timing is very similar to that of a Rhol GEF Tus1 (Yoshida et al. 2006). However, unlike type II myosin (Myol) or tropomyosin (Tpm2), which are lost from the bud neck after CAR constriction, GFP-Rhol remained concentrated at the bud neck even after mitotic exit, lasting until cells complete abscission and cell separation. Thus, live cell imaging suggests that, in addition to its role in CAR assembly Rhol may also function during late cytokinesis.

In contrast, with Rhol recrutiment during anaphase, the recruitment of Rhol to the bud neck after mitotic exit did not require GEFs or nucleotide exchange on Rho1. As expected, GFP-Rhol failed to concentrate at the bud neck during anaphase in $\triangle G E F \triangle G A P$ cells (Fig. 4B). However, GFP-Rhol was still recruited to the bud neck during mitotic exit: It was detectable at the bud neck shortly after spindle disassembly, $(n=12)$. Likewise, the GTPlocked variant GFP-Rho1-Q68H, was not recruited to the bud neck during anaphase regardless of the presence of GEFs (Fig. 4B). However, after mitotic exit GFP-Rho1$\mathrm{Q} 68 \mathrm{H}$ concentrated at the bud neck like wild-type Rho1.
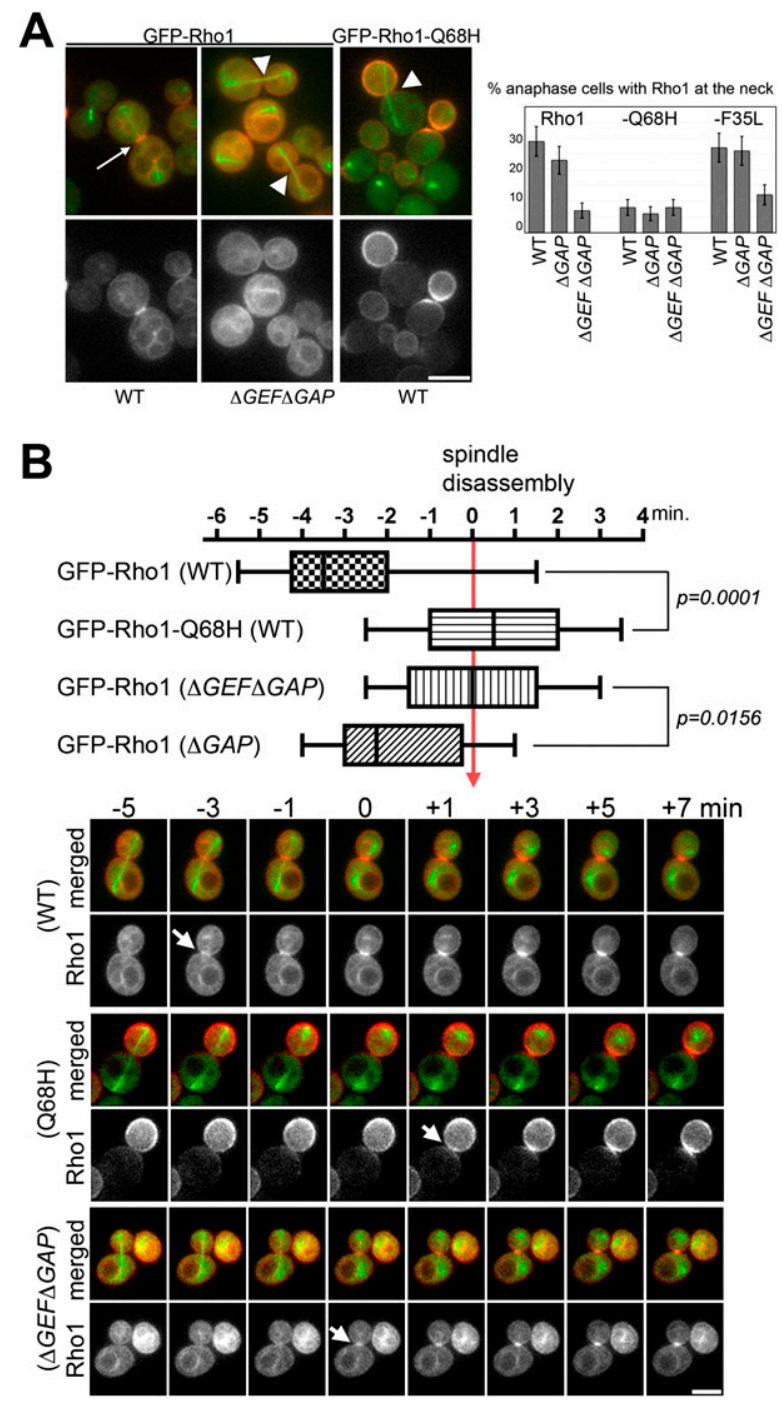

Figure 4. GEFs recruit Rhol to the division site during anaphase; a GEF-independent mechanism recruits Rhol after mitotic exit. (A) Anaphase localization of Rhol variants. Rhol mutants were expressed as GFP-fusions. Microtubules were labeled with CFP- $\alpha$-tubulin. Representative images are shown in the left. Arrows indicates the bud neck localizaton of Rhol in anaphase and arrowheads indicate the absence of Rhol. The results are summarized on the right. (B) Box plot distributions of the timing of the bud neck localization of GFP-Rhol examined by time-lapsed imaging. CFP-tubulin (in green) was used to track cell cycle position. Time of mitotic exit is judged by spindle disassembly (time $=0$ ). In these distributions, the maximum and minimum values, the interquartile range (marked by boxes), and the median value (marked by horizontal line) for the initial bud neck localization of GFP-Rhol in each population are shown. $\mathrm{CFP}$ and GFP signal were imaged every $30 \mathrm{sec}$ at $24^{\circ} \mathrm{C}$. Examples are shown at the bottom. Arrows indicate the appearance of Rhol signal at the bud neck.

Thus, GEFs are responsible for concentrating Rhol at the division site during anaphase, but an additional GEFindependent mechanism exists to concentrate Rhol at the division site after mitotic exit. 
Yoshida et al.

The PBS of Rho1 mediates GEF-independent recruitment to the division site

A subgroup of Rho GTPases contains a C-terminal PBS that binds acidic phospholipids and mediates plasma membrane targeting (Williams 2003). Because acidic phospholipids, particularly PIP2, concentrate at the division site and participate in cytokinesis in many organisms (Janetopoulos and Devreotes 2006; Logan and Mandato 2006), we considered the possibility that the GEF-independent targeting of Rhol occurred via its PBS. First, we confirmed that Rho1, like RhoA, binds acidic phospholipids. Cell extracts expressing GFP-Rhol or a variant with five lysine residues mutated to alanine (GFP-Rho1-5KA) were incubated with a lipid array (PIP Strip, Echelon) and bound proteins were detected with an anti-GFP antibody. Rhol failed to bind most membrane lipids but bound phosphorylated PIs, including PI(4)P and PI(4,5)P2 (Fig. 5A). These interactions required the basic residues of Rhol PBS (Fig. 5AB).

Next, we characterized the cell cycle pattern of localization of GFP-Rho1-5KA (Fig. 5B). As expected, GFPRho1-5KA displayed diminished plasma membrane localization. However, in control cells, GFP-Rho1-5KA was able to concentrate at the bud neck during cytokinesis (Fig. 5C). This was expected because GEFs localize to the bud neck during CAR assembly (Tus1) and after mitotic exit (Rom2). Strikingly, in the absence of Rho1 GEFs, mutation of the Rhol PBS completely abolished bud neck targeting of Rhol (Fig. 5C).
This result cannot be explained by the reduction in plasma membrane targeting of GFP-Rho1-5KA. We replaced the Rhol tail sequence, including the PBS, with the plasma membrane targeting sequence of Ras2 (Rho1$\Delta$ cRas2) (Fig. $5 \mathrm{~B}$ ). The plasma membrane targeting sequence of Ras2 is not positively charged and uniformly localizes to the plasma membrane because it is doubly prenylated (Dong et al. 2003). As expected, GFP-Rho1$\Delta \mathrm{cRas} 2$ localizes robustly to the plasma membrane and concomitantly, localization to endomembranes was barely detectable (Fig. 5C). Like GFP-Rho1-5KA, GFPRhol- $\Delta$ cRas 2 was able to concentrate at the bud neck in the presence of Rho1 GEFs. However, GFP-Rho1- $\Delta$ cRas2 failed to accumulate at the bud neck in the $\triangle G E F \triangle G A P$ strain (Fig. 5C). The reduced bud neck accumulation of GFP-Rho1-5KA and GFP-Rhol- $\Delta$ cRas2 also cannot be explained by compromised GTP loading because pulldown assays demonstrated that these constructs were loaded with GTP at comparable or increased levels relative to wild-type Rhol (Fig. 5D). Finally, defective localization of Rho1-5KA and Rhol- $\Delta$ cRas2 were not likely due to altered interaction with RhoGDI because Rhol could localize to the bud neck in $\triangle G E F \Delta$ rdi1 cells (Supplemental Fig. S3D). Together, our findings suggest that the Rhol PBS is necessary for concentrating Rhol at the division site after mitotic exit.

We next determined if the C-terminal 23-amino-acid tail of Rhol, which includes the PBS and the CAAX motif, is sufficient for bud neck targeting. We generated a fusion of GFP to the 23-amino-acid Rhol tail (GFP-Rholtail) and
Figure 5. The Rhol PBS binds lipids and is a bud neck targeting signal. (A) PIP strip (Echelon) membranes were incubated with yeast cell lysates from the cells expressing endogenous-level GFP-RHO1 or GFP-RHO1-5KA. Membrane-bound proteins were detected with an antiGFP antibody. (LPA) Lysophosphatidic acid; (LPC) lysophosphatidylcoline; (PI) phosphatidylinositol; (PE) phosphatidylethanolamine; (PC) phosphatidylcholine; (S1P) sphingosine-1-phosphate; (PA) phosphatidic acid; (PS) phosphatidylserine. $(B)$ Amino acid sequence of the Rhol tail and derivatives. Positively charged residues $(\mathrm{K})$ are in capital letters. The CAAX sequence of Rhol and plasma membrane targeting sequence of Ras2 (288322) are in italics. Mutated residues are in bold face. $(C)$ Localization of Rhol tail mutants. GFP-tagged Rhol mutants were localized in the presence $(\triangle G A P)$ or absence $(\triangle G E F \Delta G A P)$ of Rhol GEFs. Log-phase cells at $24^{\circ} \mathrm{C}$ were imaged without fixation. Bar, $3 \mu \mathrm{m}$. (D) The level of GTP-Rhol was measured in the indicated strains by a RBD pull-down assay as in Figure 2B. $(E)$ Localization of GFP-Rholtail and GFP-Ras2tail during cytokinesis. (F) GFP-Rholtail was expressed in the strain expressing septin (Shs1)-mRFP. The Rhol tail localized to the bud neck only after the septin rings split. The localization of GFP-Rholtail in cdc15-2arrested cells and after inactivation of Mss4 $13 \mathrm{~h}$, $37^{\circ} \mathrm{C}$ ) are shown. Arrows indicate accumulation to the bud neck.
A

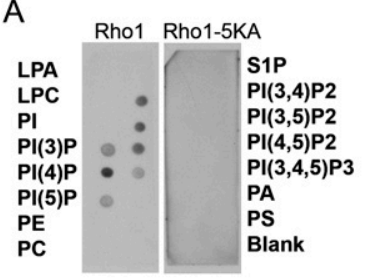

B

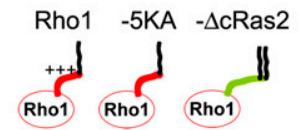

Rho1 187-KsKtngKaKKntteKKKKK-cvll209

-5KA -KsKtngKaKKntteAAAAA-cvll

- $\Delta$ CRas2 -KsKtng-288tgqvs...sgsggcciis 322
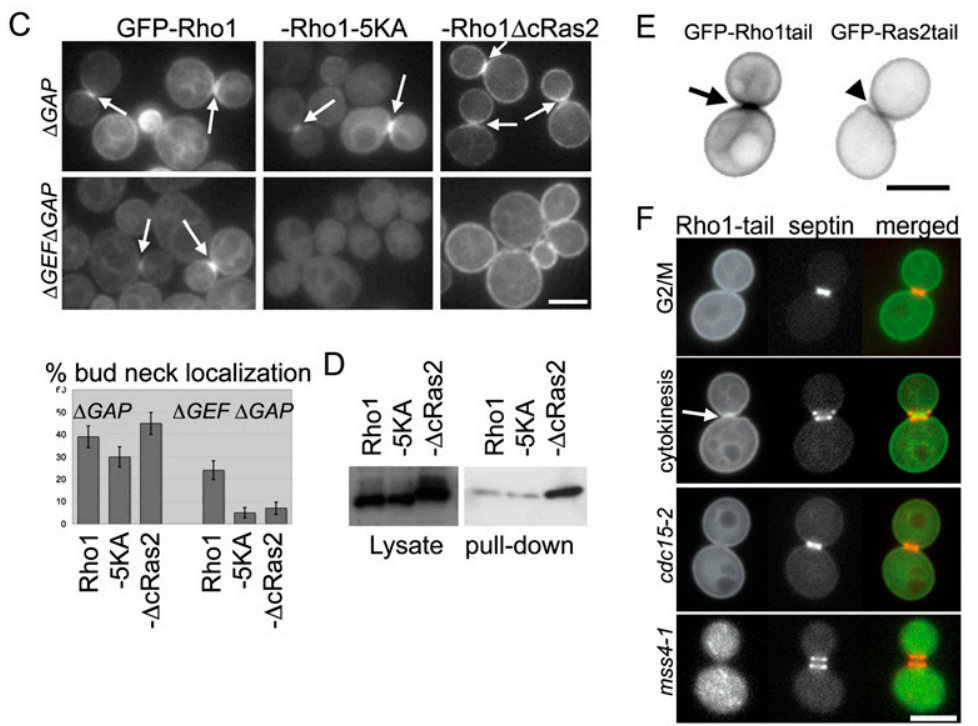
a fusion of GFP to the 34-amino-acid Ras2 tail (GFPRas2tail) (Fig. 5E). As expected from the known distribution of PIP2 in yeast, GFP-Rholtail was targeted to membranes, including the plasma membrane (Fig. 5E; Stefan et al. 2002). The plasma membrane localization of GFP-Rholtail was largely dependent on plasma membrane PIP2 because this localization was significantly impaired after inactivation of Mss4, the only yeast PI/4)P 5-kinase (Fig. 5F). Strikingly, compared with uniform localization of GFP-Ras2tail to the plasma membrane, GFP-Rhol tail became obviously enriched at the bud neck (Fig. 5E), only in late mitotic cells that had completed mitotic exit and contained split septin rings (Fig. 5F). Septins form an hourglass-shaped ring at the bud neck during polarized bud growth, and this ring splits into two rings upon mitotic exit to facilitate cytokinesis (Lippincott et al. 2001). Split septin rings create a unique membrane microdomain because of the diffusion barrier properties of septins (Dobbelaere and Barral 2004). Detailed examination revealed that GFP-Rholtail accumulates specifically between the split septin rings (Fig. 5E). In contrast, we never detected GFP-Rholtail accumulation at the bud neck in cells that contained an intact septin ring $(n=29)$.

The following evidence suggested that septin ring splitting is indeed a prerequisite for the accumulation of GFP-Rhol tail at the bud neck. First, bud neck targeting of GFP-Rhol tail was abolished after inactivation of CDC12, which encodes one of the essential yeast septins. $(1 \mathrm{~h}$ after shifting a $c d c 12-6$ mutant to $37^{\circ} \mathrm{C}, 4.8 \%$ of wild-type cells exhibited bud neck localized GFP-Rholtail vs. $0.4 \%$ of cdc12-6 cells, $n=500$ ). Next we blocked cells just prior to mitotic exit and prior to septin ring splitting by inactivation of Cdc15. In cdc15-2-arrested cells, full-length Rho1 could be recruited to the bud neck but only if Rhol GEFs were present (Yoshida et al. 2006). In contrast, GFPRholtail failed to accumulate at the bud neck in $c d c 15$ arrested cells (Fig. 5E). This result was due to the intact septin ring not to cell cycle arrest: GFP-Rholtail still failed to localize to the neck in $c d c 15$ cells after overexpression of the Cdk inhibitor Sic1, which forces mitotic exit but does not allow septin ring splitting (Lippincott et al. 2001). Thus, the Rhol tail is targeted to the membrane microdomain formed between split septin rings after mitotic exit.

The Rho1 PBS is necessary for cytokinesis in the cells lacking Rho1 GEFs.

Next, we determined if mutations in the Rhol PBS impaired cytokinesis. In an otherwise wild-type background, cells expressing only Rhol variants lacking the PBS had no detectable growth defect or defect in cytokinesis and properly assembled CAR (Fig. 6A,B; data not shown). This was expected because of our data showing that the presence of GEFs overrides a requirement for the PBS in targeting Rhol to the division site (Fig. 5C). However, a functional role for the Rhol PBS was revealed in cells lacking Rom2. $\Delta$ rom 2 rho1- $\Delta c$ Ras2 double mutant cells displayed a synthetic growth defect relative to the single
A
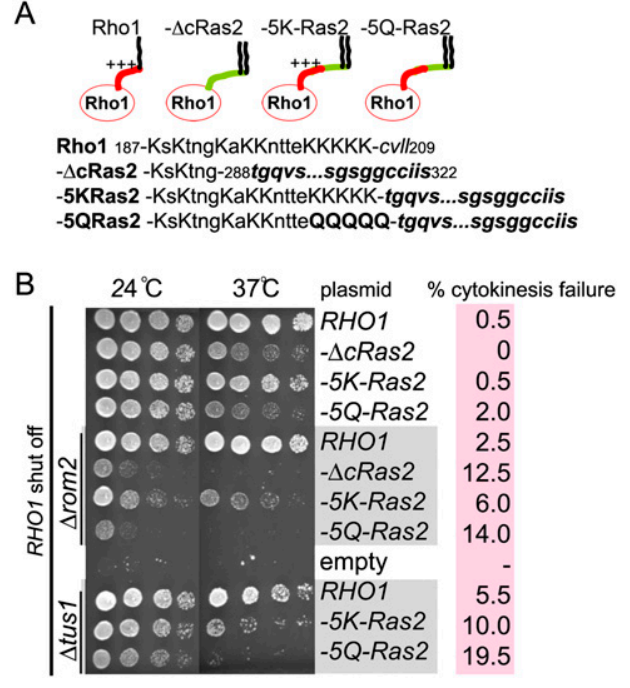

Figure 6. The Rhol PBS is necessary for normal Rhol function including cytokinesis. (A) Amino acid sequence of Rhol tail and its mutants as in Figure 5B. $(B)$ The functional importance of the basic residues in the Rhol tail for growth and cytokinesis. Strains of the indicated genotype were spotted onto plates in serial dilutions. Wild-type RHO1, expressed from the GAL1 promoter, was repressed by growth on medium containing glucose. Images were taken after $3 \mathrm{~d}$ growth at the indicated temperature. Cytokinesis failure (more than three connected cells, $n=200$ ) was scored after depletion of wild-type Rhol for $20 \mathrm{~h}$ in glucose-containing medium at $24^{\circ} \mathrm{C}$. Cells were sonicated after fixation.

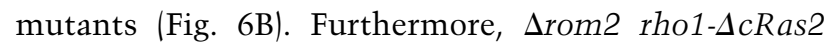
cells had a severe defect in cytokinesis (Fig. 6B). These results reflect a specific requirement for the PBS: A Rho1-Ras2 tail fusion containing a PBS (Rho1-5KRas2) enabled nearly normal growth in cells lacking Rom2, whereas a control construct where the basic lysine residues were mutated to glutamine (Rho1-5QRas2) did not. The Rho1-5KRas2-expressing cells were proficient for both growth and in cytokinesis whereas, in the absence of Rom2, the Rho1-5QRas2-expressing cells were not. A similar requirement for the Rhol PBS was apparent in cells lacking Tus1 (Fig. 6B). Therefore, reduction of Rho1GEF activity in cells reveals a functional role for the Rhol PBS in cytokinesis. Importantly, our analysis of the Ras2 tail fusions demonstrates that this functional role of the PBS extends beyond plasma membrane targeting.

\section{Functional role for Rho1 and PIP2 in CAR-independent cytokinesis}

Budding yeast utilize at least two genetically separable mechanisms to accomplish cytokinesis: a CAR-dependent mechanism that guides membrane constriction and promotes the formation of a primary septum by recruiting chitin synthase II Chs 2 to the division site (M. Schmidt et al. 2002). A less well-understood mechanism enables abscission and septation in the absence of a contractile ring. CARindependent cytokinesis is mediated by the formation of 
a secondary septum composed of chitin, assembled by chitin synthase III (Chs3) (M. Schmidt et al. 2002) and of glucan, assembled by the glucan synthases including Fks1 (Lesage and Bussey 2006). Although Rhol is known to be required for contractile ring assembly, a specific role in CAR-independent cytokinesis has not been defined. Our genetic analysis (below) demonstrated a role for Rhol in CAR-independent cytokinesis and demonstrated that this role requires the Rho1 PBS and PIP2.

We found previously that cells lacking Myol (that cannot assemble the CAR/ were not viable if they also lacked the Rho1 GEF Rom2 (Yoshida et al. 2006). This suggests that Rhol activity is essential for CAR-independent (Myol-independent) cytokinesis. Consistent with this hypothesis, we found that the synthetic lethality of a $\Delta$ myo1 $\Delta$ rom2 strain was rescued by expression of a GTP-locked Rho1-Q68H (Supplemental Fig. S4A). Most strikingly, GTP-locked RholQ68H bypassed the lethality of a $\Delta$ myo1 strain at $37^{\circ} \mathrm{C}$ (although MYO1 is not essential in our strains at $24^{\circ} \mathrm{C}$, it is essential at $37^{\circ} \mathrm{C}$ ) (Fig. 7A). Likewise, overexpression of wildtype Rhol from a $2 \mu$ plasmid also suppressed a $\Delta$ myo1 strain at $37^{\circ} \mathrm{C}$ (data not shown). Furthermore, an activated allele of the Rhol effector Pkc1 (protein kinase C in yeast) (Nonaka et al. 1995) or overexpression of the PI(4) 5 kinase Mss4 also partially restored the growth of $\Delta$ myol cells at $37^{\circ} \mathrm{C}$ (Fig. $\left.7 \mathrm{~A}\right)$. Myol is absolutely essential for CAR assembly (Bi et al. 1998), and as expected, neither RHO1-Q68H nor MSS4 restored the CAR assembly in $\triangle$ myo1 cells (Fig. 7B). Thus, Rho1, together with PIP2, activates the CAR-independent pathway for cytokinesis.

Next we tested whether Rhol and Pkcl are necessary for normal secondary septum formation, which is required for CAR-independent cytokinesis (M. Schmidt et al. 2002). Rhol and Pkcl are known to promote the transport of Chs3 from an intracellular pool to the plasma membrane (Valdivia and Schekman 2003). We therefore determined if Rhol is necessary to recruit Chs3 to the division site, an essential event for the formation of a normal secondary septum. Whereas Chs 3 accumulates at the bud neck during cytokinesis in wild-type cells (Lesage and Bussey 2006), it failed to accumulate in rho1-2 cells at $37^{\circ} \mathrm{C}$ (Fig. $7 \mathrm{C}$ ). Thus, Rhol has at least one important role in regulating secondary septum formation.

Multiple mechanisms may contribute to CAR-independent cytokinesis, and Rhol likely regulates several of these pathways. The formin Bnil, which is also important for CAR assembly (Vallen et al. 2000; Tolliday et al. 2002), and Fks1, a catalytic subunit of glucan synthase, are both required for the CAR-independent pathway of cytokinesis because $\Delta$ myo $1 \Delta b n i 1$ and $\Delta$ myo1 $\Delta f k s 1$ strains are inviable (Supplemental Fig. S4B). Furthermore, the ability of Rhol to promote CAR-independent cytokinesis requires these downstream effectors. In contrast with strains lacking only Myo1 (Amyo1) or both Myol and

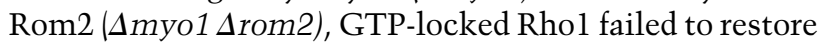
viability or effective cytokinesis to strains lacking both Myo1 and Chs3 (Fig. 7D). In addition, GTP-locked Rho1 failed to rescue $\Delta$ myo1 $\Delta b n i 1$ or $\Delta$ myo1 $\Delta$ fks 1 strains (Supplemental Fig. S4).

Altogether, our work demonstrates that Rhol promotes cytokinesis in several ways: It has a critical role in CAR assembly, and the above results suggest it can promote CAR independent cytokinesis through effects on local chitin synthesis and cell wall assembly.

\section{Discussion}

The concentration and local activation of the small GTPase Rho at the division site is a general feature of cytokinesis in organisms as divergent as yeasts and metazoans. This "active Rho zone" is essential for cleavage furrow formation and the position of the active Rho zone determines the position of the furrow (Bement et al. 2005; Piekny et al. 2005). Here we comprehensively characterized the mechanisms that concentrate Rho1 (RhoA) at the division site in budding yeast. We demonstrated the existence of a GEF-dependent mechanism that requires that Rhol has the ability to pass through the

Figure 7. Rhol promotes CAR-independent cytokinesis. (A) GTP-locked Rhol, an activated mutant of Pkc1, or overexpression of the PI(4)P 5-kinase Mss4 rescues growth defect of $\Delta m y o 1$ at $37^{\circ} \mathrm{C}$. $(B)$ Neither GTP-locked Rho1 nor Mss4 restore CAR assembly in $\Delta$ myo1 strains. The indicated strains were fixed and stained with Alexa-568-conjugated phalloidin. The percentage of large budded cells with detectable CAR assembly was scored. $(C)$ The bud neck localization of Chs3 requires Rhol activity. Localization of Chs3-GFP was monitored after Rhol inactivation $(2 \mathrm{~h}$ at $37^{\circ} \mathrm{C}$ ). Mitotic spindle visualized by GFP-tubulin is used as a cell cycle marker. The percentage of late mitotic cells with Chs 3 accumulation to the neck was scored after fixation. (D) GTP-locked Rho1 requires CHS3 to suppress the growth defect of $\Delta$ myo1. A plasmid shuffle was performed as in $A$ for

A

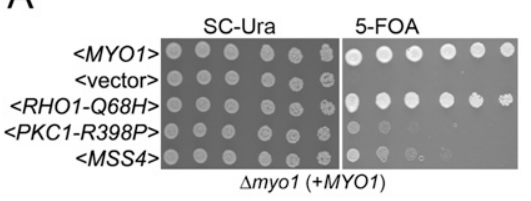

B

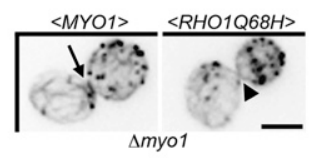

$\%$ CAR assembly in $\Delta m y 01$

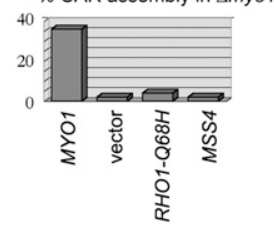

C

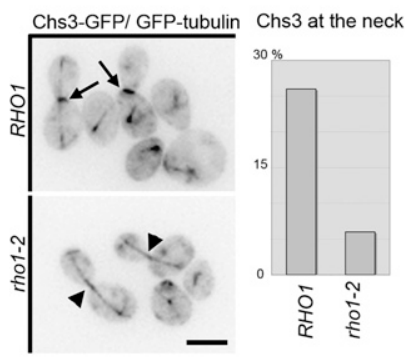

D

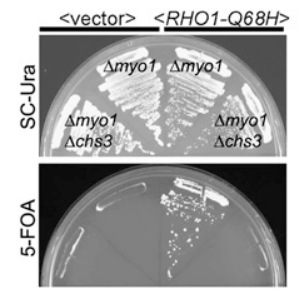


GDP-liganded state to enable CAR assembly. Our findings also revealed a GEF-independent mechanism that functions after mitotic exit. This mechanism requires PIP2, and depends on an intact membrane microdomain formed by split septin rings.

The requirement for RhoGEFs and Rho GTPase cycle to create an active Rho zone

We previously reported that CAR assembly in budding yeast is triggered by the Polo-like kinase Cdc5. During anaphase Cdc5/Polo, binds, and phosphorylates Rho1 GEFs, most importantly for CAR assembly Tus1. Phosphorylated Tus1 is targeted to the bud neck in late anaphase where it facilitates Rhol targeting and activation (Yoshida et al. 2006). Targeting of Tus1 is a critical regulatory step because the requirement of $\mathrm{Cdc} 5 /$ Polo for CAR assembly can be partially bypassed by fusing Tus1 to a bud neck resident protein. If Rhol GEFs are not recruited to the division site, Rhol itself fails to concentrate at the division site.

The role of Tus1 is not simply to activate Rhol. Surprisingly, we found that GTP-locked Rhol could not bypass the requirement for Cdc5 or Rhol GEFs for CAR assembly. This was explained by the fact that GTP-locked Rhol was unable to concentrate at the division site in anaphase. This suggested the appealing hypothesis that Rho GEFs, through their binding to GDP-Rhol were directly responsible for the initial recruitment of Rho to the division site. Our ability to engineer viable yeast strains lacking all Rhol GEFs enabled a direct test of this model and allowed us to establish that the GEF requirement reflected a requirement for RhoGEF catalytic activity.

Although binding of GDP-Rhol to the DH domain of Tus1 (and other GEFs) appears to be crucial for the initial recruitment of Rhol, in the cell, binding will stimulate GTP exchange and dissociation of Rhol from the GEF. This dissociation is expected to be rapid based on the halflife of Rho-GEF complexes in the presence of physiological concentrations of GTP. GTP-Rhol has key effectors necessary for cytokinesis such as the formin Bnil that catalyzes actin filament assembly during CAR assembly. We suggest that Rho1 binds effectors after release from its GEF; effector binding is therefore likely to be important for maintaining Rhol at the division site after its initial recruitment. Identification of all of the Rhol effectors at the division site and determining which ones (or combinations) might be important for Rho1 maintenance at the neck will be an interesting direction for future work.

The GEF-dependent Rho recruitment and the requirement for Rho cycling might be general features of cytokinesis. In animal cells, inhibition of Polo results in a failure to recruit both RhoA GEFs and RhoA to the cell equator (Takaki et al. 2008). Our work raises the attractive possibility that this reflects a direct role for the $\mathrm{DH}$ domains of Rho GEFs to recruit GDP-RhoA. RhoA GTPase flux is also likely to affect the equatorial concentration of active Rho in other ways. Mathematical modeling suggests that active RhoA could accumulate at the cell equator by a reaction-diffusion mechanism requiring GAP-dependent RhoA inactivation (Bement et al. 2006). Supporting this idea, recent analysis of mutants lacking the GAP activity of MgcRacGAP reveals diffuse, disorganized zones of active RhoA (Miller and Bement 2009).

\section{Is nucleotide exchange on Rho1 an essential function in budding yeast?}

We find that budding yeast can survive in the absence of all recognizable Rhol GEFs if compensatory mutations in GAPs are present in the cell. This raises the question of whether exchange is an essential function in yeast or whether there exist other, not yet recognized, mediators of nucleotide exchange for Rho1. Spontaneous GDP release by Rhol is very slow (Ozaki et al. 1996; Schmelzle et al. 2002). It is possible that newly synthesized Rho1 binds GTP and in cells that have diminished GAP function, the GTP-loaded Rhol present in cells can support viability. Although exchange is important for CAR assembly, the CAR is not essential in budding yeast. Furthermore, GTP-locked Rhol can promote certain essential Rhol functions such as the activation of glucan synthesis (Sekiya-Kawasaki et al. 2002). Thus, it is possible that exchange is not absolutely essential in budding yeast. Alternatively, there may be unrecognized exchange mechanisms. Both in vitro and in vivo evidence make it very unlikely that the Cdc 42 exchange factor Cdc24 acts on Rho1 (Park and Bi 2007). Another possible Rho1 GEF is Ylr422wp, which belongs to a recently identified family of proteins (CZH proteins) some of which can act as RhoGEFs (Meller et al. 2005). However, deletion of YLR422 $w$ does not compromise the growth of $\triangle G E F \triangle G A P$ strains (S. Yoshida, unpubl.), nor does it have any other known genetic interactions with Rhol pathway mutations. Finally, it remains possible that there exist other exchange mechanisms that could be identified by genetic suppression of triple GEF deletion strains.

\section{GEF-independent Rho1 targeting during late stages of cytokinesis}

Our analysis of strains lacking Rhol GEFs revealed the existence of a GEF-independent mechanism for concentrating Rhol at the division site. This GEF-independent mechanism requires the Rho1 C-terminal PBS, PIP2, and functional septins. GEF-independent recruitment occurs only after mitotic exit when a membrane microdomain forms, bounded by split septin rings. The following evidence supports the idea that the PBS of Rhol interacts with PIP2 in vivo: Like other family members, the PBS of yeast Rho1 is required for PIP2 binding in vitro; the localization of the Rho1 PBS (C-terminal 23 amino acids) requires PI(4)P 5-kinase activity (Mss4); and increased cellular PIP2 can promote cytokinesis in the absence of a contractile ring. The concentration of PIP2 is enriched at the division site in many eukaryotes and PIP2 is required for cytokinesis completion in many cell types (Janetopoulos and Devreotes 2006; Logan and Mandato 2006). Indeed, using a GFP-PH domain reporter, PIP2 recently has been found to concentrate at the bud neck 
in budding yeast (C. Stefan and S. Emr, pers. comm.). Because the GEF-independent recruitment of Rhol requires septins and yeast septins bind to membrane phospholipids (Casamayor and Snyder 2003), it is attractive to postulate that the enrichment of PIP2 at the bud neck is mediated by septins. However, our data do not exclude the possibility that the PBS sequence of Rhol also interacts with another membrane lipid or protein.

GEF-independent localization appears to be a backup pathway that becomes essential in cells lacking Rhol GEFs. In wild-type cells, the Rho1 GEF Rom2 remains at the bud neck during mitotic exit, and thus it is expected that GEF-dependent recruitment of Rhol will, in normal circumstances, occur after mitotic exit. However, in the absence of GEFs, the PBS becomes essential for concentrating Rhol at the bud neck, even if plasma membrane localization is forced by fusion to the doubly prenylated Ras2 tail. The Rho1 GEF Rom2 is likely to utilize a similar mechanism to remain at the bud neck after mitotic exit because of the interaction between the Rom 2 PH domain and PIP2 (Audhya and Emr 2002). The combination of $\mathrm{PH}$ domain-dependent localization of Rom2 and the PBS-dependent localization of Rhol nicely explains how Rhol can be maintained at the division site even after Cdc5/Polo kinase is degraded during mitotic exit (Lee et al. 2005).

PIP2 concentrates at the division site in many organisms. The molecular function of PIP2 in cytokinesis is poorly understood, but is likely to be complex. PIP2 is not required for CAR assembly, but is essential for completion of cytokinesis after furrow ingression (Janetopoulos and Devreotes 2006; Logan and Mandato 2006). PIP2 regulates both endocytosis and exocytosis and both processes are required for executing the late stages of cytokinesis (Barr and Gruneberg 2007). Interestingly, a recent study showed that a lipid-binding protein Anillin has a role in maintaining RhoA at the division site during late stage cytokinesis in human cells (Piekny and Glotzer 2008). Based on our results, we propose that one way that PIP2 promotes cytokinesis is to facilitate the local accumulation of Rho GTPases and their GEFs.

Our findings also have implications for the regional organization of the plasma membrane. Small GTPases that localize to the plasma membrane either contain multiple sites of lipid modification or have a single site of lipid modification and a PBS (Heo et al. 2006). Functional differences between these classes of GTPases are only just being defined. However, the K-Ras PBS appears to be important for its segregation into plasma membrane nanoclusters that are distinct from clusters containing $\mathrm{H}$ Ras (Plowman et al. 2008). Here we demonstrated that the C-terminal PBS domain of Rhol is necessary and sufficient for targeting to a specific plasma membrane region: the membrane microdomain formed by split septin rings.

\section{Multiple roles for Rho1 during cytokinesis}

Our results demonstrate a functional requirement for the Rho1 PBS and PIP2 in cytokinesis in budding yeast, specifically for late-stage, CAR-independent cytokinesis.
Consistent with the GEF-independent localization of Rhol that we observed, the functional role of Rhol during CAR-independent cytokinesis does not require the ability of Rhol to cycle between the GTP and GDP-liganded states, as GTP-locked Rhol-Q68H promotes cytokinesis in $\Delta$ myo1 strains that lack CAR.

Our genetic analysis suggests that Rhol acts at multiple levels to promote CAR-independent cytokinesis. The suppression of severe growth defect of $\Delta$ myo 1 at $37^{\circ} \mathrm{C}$ by up-regulation of RHO1, MSS4, or PKC1 suggested that Rhol signaling could promote efficient cytokinesis by activating secondary septum formation. Rhol and Pkcl potentially activate both the cell wall integrity MAP kinase pathway (Mpk1) and Chs3 transport to promote secondary septum assembly. Furthermore, the synthetic lethality between $\Delta$ myol and $\Delta f k s 1$ suggests that Rhol also directly promotes septum biogenesis because the glucan synthase activity of Fks1 depends on Rhol. Supporting their role in MYO1-independent function in cytokinesis, $\Delta$ myo1 $\Delta m p k 1$ double mutant strains were lethal and $\Delta$ myo1 $\Delta$ chs3 strain displayed a synthetic growth defect (data not shown), consistent with other recent reports (M. Schmidt et al. 2002; RodriguezQuinones et al. 2008).

In addition to septum assembly, Rhol may play a role in abscission by promoting secretion. Activated Rhol binds and controls the localization of a landmark protein for secretion Sec3 (Guo et al. 2001), which is required for abscission in budding yeast (Dobbelaere and Barral 2004; VerPlank and Li 2005). Bnil is a formin protein required both for CAR assembly and normal septin assembly. Although the requirement for Bnil in the CAR-dependent pathway has been described (Vallen et al. 2000; Tolliday et al. 2002), a role for Bnil in CAR-independent cytokinesis is novel. Bnil is the only formin localized at the division site during cytokinesis (Buttery et al. 2007). A plausible way that Bnil could affect CAR-independent cytokinesis would be through actin cable assembly and polarized secretion. It is important to note that both Chs 2 (required for primary septum formation) and Chs3 (required for secondary septum formation) are targeted to the division site by secretion after mitotic exit (Dobbelaere and Barral 2004; VerPlank and Li 2005; Zhang et al. 2006). Thus, Rhol is a master regulator of budding yeast cytokinesis. Rhol promotes CAR assembly and thus primary septum formation. Rhol also promotes secondary septum formation and abscission through effects on cell wall biogenesis and secretion (Fig. 8).

\section{Summary}

We defined two mechanisms for concentrating Rhol at the division site in budding yeast, both of which are functionally important for cytokinesis. The GEF-dependent mechanism is likely to be relevant to cytokinesis in animal cells because RhoA GEFs are required for concentrating RhoA at the cleavage site in many organisms. The GEF-independent mechanism may also be generally relevant because PIP2 is enriched at the division site in many cell types. A direct interaction between the RhoA 


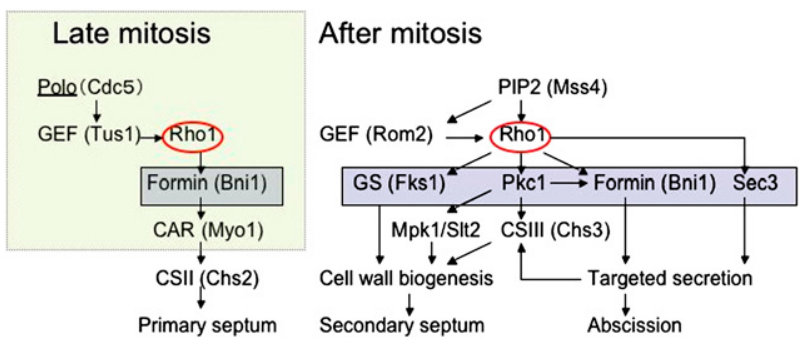

Figure 8. A model of Rhol regulation and function in cytokinesis. (Left) CAR assembly in late anaphase. Polo-dependent recruitment of Tus1 promotes CAR assembly by recruiting Rhol to the division site. After mitotic exit, Chs2 is transported to the bud neck and CAR constriction guides primary septum formation. (Right) After mitotic exit, Polo is degraded and PIP2 plays an important role in concentrating Rhol activity at the bud neck. See the Discussion for details. (GS) Glucan synthase; (CS) chitin synthase.

PBS and PIP2 could contribute to maintaining the pool of RhoA that participates in late events in cytokinesis.

\section{Materials and methods}

\section{Yeast genetics and strains}

All yeast strains (except for SY1546 and SY1548) were isogenic or congenic to BY4741 (MATa his3 leu2 met15 ura3 from Open Biosystems). Gene deletion or modifications were performed with PCR-mediated gene replacement, and accurate integration was confirmed by colony PCR. For a description of the strains and plasmids, see Supplemental Tables S1 and S2.

\section{Fluorescence imaging and image analysis}

An automated Zeiss 200M inverted microscope (Carl Zeiss) was used for imaging as described (Buttery et al. 2007). All image processing was performed using SlideBook software. To characterize the localization of proteins to the bud neck, we acquired images at $0.5-\mu \mathrm{m}$ intervals in the $\mathrm{Z}$ focal plane, enabling visualization of the relatively small budding yeast bud neck region (usually $<1 \mu \mathrm{m}$ in diameter). For time-lapse live cell microscopy $2 \mu \mathrm{L}$ of the culture (in the synthetic complete [SC] medium supplemented with adenine) were spotted onto a glass slide and immediately imaged at room temperature $\left(\sim 24^{\circ} \mathrm{C}\right)$. The immunofluorescence procedure for Rhol and HA-Rhol was described previously (Abe et al. 2003).

\section{Biochemical methods}

Mouse monoclonal anti-myc antibody (9E10), mouse monoclonal anti-HA antibody (12CA5), rabbit polyclonal anti-Mpk1/Slt2 antibody, and mouse monoclonal anti-GFP antibody were obtained from commercial sources. Rabbit anti-Rhol antibody was described previously (Abe et al. 2003). Western blotting and the pull-down assay for active Rholp was performed as described (Yoshida et al. 2006; Kono et al. 2008).

\section{In vitro lipid-binding assay}

GFP-Rhol-containing yeast extracts were prepared by bead beating in lysis buffer $(50 \mathrm{mM}$ Tris at $\mathrm{pH} 8.0 ; 10 \mathrm{mM}$ EDTA at
$\mathrm{pH} 8.0 ; 100 \mathrm{mM} \mathrm{NaCl}$; 0.5\% Triton X-100; protease inhibitor cocktail). Each PIP Strip was preincubated for $1 \mathrm{~h}$ in $3 \%$ fattyacid-free BSA in TBS-T. Preincubated PIP strips were incubated with cell lysate in $3 \%$ BSA in TBS-T with gentle shaking overnight. After washing three times with $1 \%$ BSA in TBS-T, the strips were incubated with anti-GFP antibody $(1: 1000)$ in $1 \%$ BSA in TBS-T for an hour. After washing three times with $1 \%$ BSA in TBS-T, the strips were incubated with anti-rabbit IgG conjugated with HRP.

\section{Acknowledgments}

We thank Y. Ohya, M. Hall, and J. Pringle for the gift of strains and plasmids; Riki Eggert for valuable comments on the manuscript; and K. Kono, B. Atkins, and other members of Pellman laboratory for discussion. This work was supported by an NIH grant to D.P. S.Y. was supported by a JSPS Fellowship and by a Charles A. King Trust Fellowship. D.P. is an investigator of Howard Hughes Medical Institute.

\section{References}

Abe, M., Qadota, H., Hirata, A., and Ohya, Y. 2003. Lack of GTPbound Rholp in secretory vesicles of Saccharomyces cerevisiae. J. Cell Biol. 162: 85-97.

Audhya, A. and Emr, S.D. 2002. Stt4 PI 4-kinase localizes to the plasma membrane and functions in the Pkc1-mediated MAP kinase cascade. Dev. Cell 2: 593-605.

Ayscough, K.R., Eby, J.J., Lila, T., Dewar, H., Kozminski, K.G., and Drubin, D.G. 1999. Slalp is a functionally modular component of the yeast cortical actin cytoskeleton required for correct localization of both Rholp-GTPase and Sla2p, a protein with talin homology. Mol. Biol. Cell 10: 1061-1075.

Balasubramanian, M.K., Bi, E., and Glotzer, M. 2004. Comparative analysis of cytokinesis in budding yeast, fission yeast and animal cells. Curr. Biol. 14: R806-R818. doi: 10.1016/ j.cub.2004.09.022.

Barr, F.A. and Gruneberg, U. 2007. Cytokinesis: Placing and making the final cut. Cell 131: 847-860.

Bement, W.M., Benink, H.A., and von Dassow, G. 2005. A microtubule-dependent zone of active RhoA during cleavage plane specification. J. Cell Biol. 170: 91-101.

Bement, W.M., Miller, A.L., and von Dassow, G. 2006. Rho GTPase activity zones and transient contractile arrays. Bioessays 28: 983-993.

Bi, E., Maddox, P., Lew, D.J., Salmon, E.D., McMillan, J.N., Yeh, E., and Pringle, J.R. 1998. Involvement of an actomyosin contractile ring in Saccharomyces cerevisiae cytokinesis. I. Cell Biol. 142: 1301-1312.

Buttery, S.M., Yoshida, S., and Pellman, D. 2007. Yeast formins Bnil and Bnrl utilize different modes of cortical interaction during the assembly of actin cables. Mol. Biol. Cell 18: 1826-1838.

Casamayor, A. and Snyder, M. 2003. Molecular dissection of a yeast septin: Distinct domains are required for septin interaction, localization, and function. Mol. Cell. Biol. 23: 2762-2777.

Delley, P.A. and Hall, M.N. 1999. Cell wall stress depolarizes cell growth via hyperactivation of RHO1. J. Cell Biol. 147: 163-174.

Dobbelaere, J. and Barral, Y. 2004. Spatial coordination of cytokinetic events by compartmentalization of the cell cortex. Science 305: 393-396.

Dong, X., Mitchell, D.A., Lobo, S., Zhao, L., Bartels, D.J., and Deschenes, R.J. 2003. Palmitoylation and plasma membrane localization of Ras 2 p by a nonclassical trafficking pathway in Saccharomyces cerevisiae. Mol. Cell. Biol. 23: 6574-6584. 
Eitzen, G., Thorngren, N., and Wickner, W. 2001. Rholp and $\mathrm{Cdc} 42 \mathrm{p}$ act after $\mathrm{Ypt} 7 \mathrm{p}$ to regulate vacuole docking. EMBO J. 20: $5650-5656$.

Fujiwara, T., Bandi, M., Nitta, M., Ivanova, E.V., Bronson, R.T., and Pellman, D. 2005. Cytokinesis failure generating tetraploids promotes tumorigenesis in p53-null cells. Nature 437: 1043-1047.

Guo, W., Tamanoi, F., and Novick, P. 2001. Spatial regulation of the exocyst complex by Rhol GTPase. Nat. Cell Biol. 3: 353360.

Heo, W.D., Inoue, T., Park, W.S., Kim, M.L., Park, B.O., Wandless, T.J., and Meyer, T. 2006. PI(3,4,5)P3 and PI(4,5)P2 lipids target proteins with polybasic clusters to the plasma membrane. Science 314: 1458-1461.

Janetopoulos, C. and Devreotes, P. 2006. Phosphoinositide signaling plays a key role in cytokinesis. J. Cell Biol. 174: 485-490.

Kimura, K., Tsuji, T., Takada, Y., Miki, T., and Narumiya, S. 2000. Accumulation of GTP-bound RhoA during cytokinesis and a critical role of ECT2 in this accumulation. J. Biol. Chem. 275: 17233-17236.

Knaus, M., Pelli-Gulli, M.P., van Drogen, F., Springer, S., Jaquenoud, M., and Peter, M. 2007. Phosphorylation of Bem $2 p$ and Bem3p may contribute to local activation of Cde42p at bud emergence. EMBO J. 26: 4501-4513.

Kono, K., Nogami, S., Abe, M., Nishizawa, M., Morishita, S., Pellman, D., and Ohya, Y. 2008. G1/S cyclin-dependent kinase regulates small GTPase Rholp through phosphorylation of RhoGEF Tuslp in Saccharomyces cerevisiae. Mol. Biol. Cell 19: 1763-1771.

Lee, K.S., Park, J.E., Asano, S., and Park, C.J. 2005. Yeast pololike kinases: Functionally conserved multitask mitotic regulators. Oncogene 24: 217-229.

Lesage, G. and Bussey, H. 2006. Cell wall assembly in Saccharomyces cerevisiae. Microbiol. Mol. Biol. Rev. 70: 317-343.

Lin, R., Bagrodia, S., Cerione, R., and Manor, D. 1997. A novel Cdc42Hs mutant induces cellular transformation. Curr. Biol. 7: 794-797.

Lippincott, J., Shannon, K.B., Shou, W., Deshaies, R.J., and Li, R. 2001. The Teml small GTPase controls actomyosin and septin dynamics during cytokinesis. J. Cell Sci. 114: 13791386.

Logan, M.R. and Mandato, C.A. 2006. Regulation of the actin cytoskeleton by PIP2 in cytokinesis. Biol. Cell 98: 377-388.

Lorberg, A., Schmitz, H.P., Jacoby, J.J., and Heinisch, J.J. 2001. Lrglp functions as a putative GTPase-activating protein in the Pkclp-mediated cell integrity pathway in Saccharomyces cerevisiae. Mol. Genet. Genomics 266: 514-526.

Manning, B.D., Padmanabha, R., and Snyder, M. 1997. The RhoGEF Rom2p localizes to sites of polarized cell growth and participates in cytoskeletal functions in Saccharomyces cerevisiae. Mol. Biol. Cell 8: 1829-1844.

Meller, N., Merlot, S., and Guda, C. 2005. CZH proteins: A new family of Rho-GEFs. J. Cell Sci. 118: 4937-4946.

Miller, A.L. and Bement, W.M. 2009. Regulation of cytokinesis by Rho GTPase flux. Nat. Cell Biol. 11: 71-77.

Nonaka, H., Tanaka, K., Hirano, H., Fujiwara, T., Kohno, H., Umikawa, M., Mino, A., and Takai, Y. 1995. A downstream target of RHO1 small GTP-binding protein is $\mathrm{PKCl}$, a homolog of protein kinase $\mathrm{C}$, which leads to activation of the MAP kinase cascade in Saccharomyces cerevisiae. EMBO J. 14: 5931-5938.

Ozaki, K., Tanaka, K., Imamura, H., Hihara, T., Kameyama, T., Nonaka, H., Hirano, H., Matsuura, Y., and Takai, Y. 1996. Rom 1p and Rom2p are GDP/GTP exchange proteins (GEPs) for the Rholp small GTP binding protein in Saccharomyces cerevisiae. EMBO J. 15: 2196-2207.

Park, H.O. and Bi, E. 2007. Central roles of small GTPases in the development of cell polarity in yeast and beyond. Microbiol. Mol. Biol. Rev. 71: 48-96.

Paumi, C.M., Menendez, J., Arnoldo, A., Engels, K., Iyer, K.R., Thaminy, S., Georgiev, O., Barral, Y., Michaelis, S., and Stagljar, I. 2007. Mapping protein-protein interactions for the yeast $\mathrm{ABC}$ transporter Ycflp by integrated split-ubiquitin membrane yeast two-hybrid analysis. Mol. Cell 26: 15-25.

Piekny, A.J. and Glotzer, M. 2008. Anillin is a scaffold protein that links RhoA, actin, and myosin during cytokinesis. Curr. Biol. 18: 30-36.

Piekny, A., Werner, M., and Glotzer, M. 2005. Cytokinesis: Welcome to the Rho zone. Trends Cell Biol. 15: 651-658.

Plowman, S.J., Ariotti, N., Goodall, A., Parton, R.G., and Hancock, J.F. 2008. Electrostatic interactions positively regulate K-Ras nanocluster formation and function. Mol. Cell. Biol. 28: 4377-4385.

Rodriguez-Quinones, J.F., Irizarry, R.A., Diaz-Blanco, N.L., RiveraMolina, F.E., Gomez-Garzon, D., and Rodriguez-Medina, J.R. 2008. Global mRNA expression analysis in myosin II deficient strains of Saccharomyces cerevisiae reveals an impairment of cell integrity functions. BMC Genomics 9: 34. doi: 10.1186/ 1471-2164-9-34.

Rossman, K.L., Worthylake, D.K., Snyder, J.T., Cheng, L., Whitehead, I.P., and Sondek, J. 2002. Functional analysis of cdc42 residues required for Guanine nucleotide exchange. $J$. Biol. Chem. 277: 50893-50898.

Rossman, K.L., Der, C.J., and Sondek, J. 2005. GEF means go: Turning on RHO GTPases with guanine nucleotide-exchange factors. Nature Reviews 6: 167-180.

Schmelzle, T., Helliwell, S.B., and Hall, M.N. 2002. Yeast protein kinases and the RHO1 exchange factor TUS1 are novel components of the cell integrity pathway in yeast. Mol. Cell. Biol. 22: 1329-1339.

Schmidt, A., Schmelzle, T., and Hall, M.N. 2002. The RHO1GAPs SAC7, BEM2 and BAG7 control distinct RHO1 functions in Saccharomyces cerevisiae. Mol. Microbiol. 45: 14331441.

Schmidt, M., Bowers, B., Varma, A., Roh, D.H., and Cabib, E. 2002. In budding yeast, contraction of the actomyosin ring and formation of the primary septum at cytokinesis depend on each other. J. Cell Sci. 115: 293-302.

Sekiya-Kawasaki, M., Abe, M., Saka, A., Watanabe, D., Kono, K., Minemura-Asakawa, M., Ishihara, S., Watanabe, T., and Ohya, Y. 2002. Dissection of upstream regulatory components of the Rholp effector, 1,3- $\beta$-glucan synthase, in Saccharomyces cerevisiae. Genetics 162: 663-676.

Stefan, C.J., Audhya, A., and Emr, S.D. 2002. The yeast synaptojanin-like proteins control the cellular distribution of phosphatidylinositol (4,5)-bisphosphate. Mol. Biol. Cell 13: 542-557.

Symons, M. and Settleman, J. 2000. Rho family GTPases: More than simple switches. Trends Cell Biol. 10: 415-419.

Taira, K., Umikawa, M., Takei, K., Myagmar, B.E., Shinzato, M., Machida, N., Uezato, H., Nonaka, S., and Kariya, K. 2004. The Traf2- and Nck-interacting kinase as a putative effector of Rap2 to regulate actin cytoskeleton. J. Biol. Chem. 279: $49488-49496$.

Takaki, T., Trenz, K., Costanzo, V., and Petronczki, M. 2008. Polo-like kinase 1 reaches beyond mitosis-Cytokinesis, DNA damage response, and development. Curr. Opin. Cell Biol. 20: 650-660. 
Tolliday, N., VerPlank, L., and Li, R. 2002. Rho1 directs forminmediated actin ring assembly during budding yeast cytokinesis. Curr. Biol. 12: 1864-1870.

Tolliday, N., Pitcher, M., and Li, R. 2003. Direct evidence for a critical role of myosin II in budding yeast cytokinesis and the evolvability of new cytokinetic mechanisms in the absence of myosin II. Mol. Biol. Cell 14: 798-809.

Valdivia, R.H. and Schekman, R. 2003. The yeasts Rholp and Pkclp regulate the transport of chitin synthase III (Chs3p) from internal stores to the plasma membrane. Proc. Natl. Acad. Sci. 100: 10287-10292.

Vallen, E.A., Caviston, J., and Bi, E. 2000. Roles of Hof1p, Bnilp, Bnrlp, and myolp in cytokinesis in Saccharomyces cerevisiae. Mol. Biol. Cell 11: 593-611.

VerPlank, L. and Li, R. 2005. Cell cycle-regulated trafficking of Chs2 controls actomyosin ring stability during cytokinesis. Mol. Biol. Cell 16: 2529-2543.

Watanabe, D., Abe, M., and Ohya, Y. 2001. Yeast Lrg1p acts as a specialized RhoGAP regulating 1,3- $\beta$-glucan synthesis. Yeast 18: 943-951.

Williams, C.L. 2003. The polybasic region of Ras and Rho family small GTPases: A regulator of protein interactions and membrane association and a site of nuclear localization signal sequences. Cell. Signal. 15: 1071-1080.

Yamochi, W., Tanaka, K., Nonaka, H., Maeda, A., Musha, T., and Takai, Y. 1994. Growth site localization of Rhol small GTPbinding protein and its involvement in bud formation in Saccharomyces cerevisiae. J. Cell Biol. 125: 1077-1093.

Yoshida, S., Kono, K., Lowery, D.M., Bartolini, S., Yaffe, M.B., Ohya, Y., and Pellman, D. 2006. Polo-like kinase Cdc5 controls the local activation of Rhol to promote cytokinesis. Science 313: 108-111.

Yoshizaki, H., Ohba, Y., Kurokawa, K., Itoh, R.E., Nakamura, T., Mochizuki, N., Nagashima, K., and Matsuda, M. 2003. Activity of Rho-family GTPases during cell division as visualized with FRET-based probes. J. Cell Biol. 162: 223232.

Zhang, G., Kashimshetty, R., Ng, K.E., Tan, H.B., and Yeong, F.M. 2006. Exit from mitosis triggers Chs2p transport from the endoplasmic reticulum to mother-daughter neck via the secretory pathway in budding yeast. J. Cell Biol. 174: 207220.

Zhu, K., Debreceni, B., Li, R., and Zheng, Y. 2000. Identification of Rho GTPase-dependent sites in the Dbl homology domain of oncogenic Dbl that are required for transformation. J. Biol. Chem. 275: 25993-26001. 


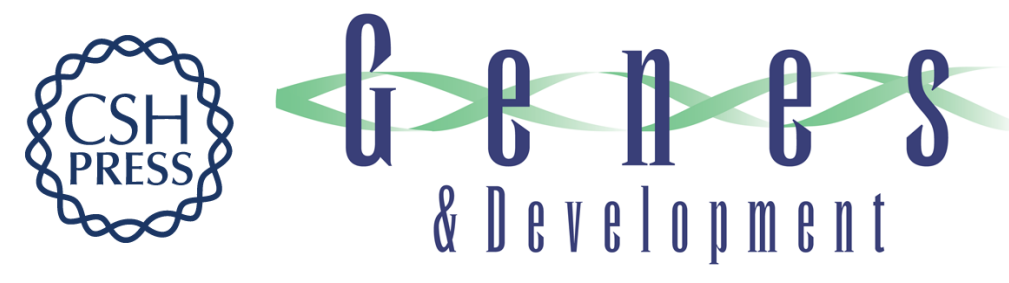

\section{Mechanisms for concentrating Rho1 during cytokinesis}

Satoshi Yoshida, Sara Bartolini and David Pellman

Genes Dev. 2009, 23:

Access the most recent version at doi:10.1101/gad.1785209

Supplemental http://genesdev.cshlp.org/content/suppl/2009/04/02/23.7.810.DC1
Material

References This article cites 60 articles, 35 of which can be accessed free at: http://genesdev.cshlp.org/content/23/7/810.full.html\#ref-list-1

License

Email Alerting Receive free email alerts when new articles cite this article - sign up in the box at the top Service right corner of the article or click here.

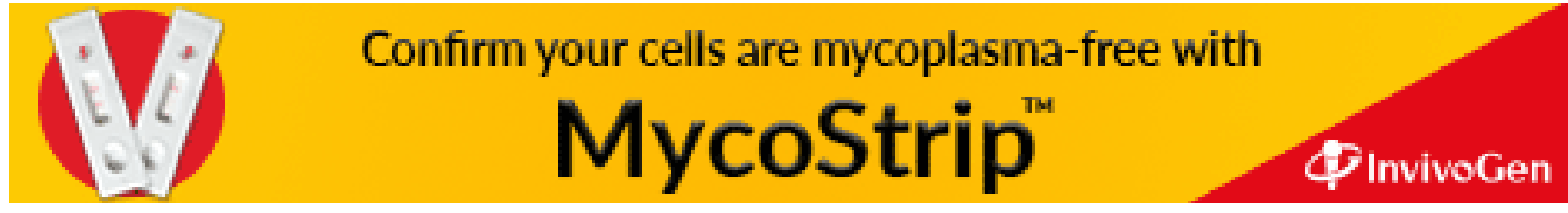

